\title{
Flexibility oriented design of a horizontal wrapping machine
}

\author{
H. Giberti ${ }^{1}$ and A. Pagani ${ }^{2}$ \\ ${ }^{1}$ Politecnico Di Milano, Dipartimento di Meccanica, Campus Bovisa Sud, via La Masa 1, 20156, Milano, Italy \\ ${ }^{2}$ Fpz S.p.a., Via Fratelli Cervi, 18, 20049 Concorezzo (MB), Italy \\ Correspondence to: H. Giberti (hermes.giberti@polimi.it)
}

Received: 17 March 2015 - Revised: 19 June 2015 - Accepted: 6 July 2015 - Published: 24 July 2015

\begin{abstract}
Flexibility and high production volumes are very important requirements in modern production lines. In most industrial processes, in order to reach high production volumes, the items are rarely stopped into a production line and all the machining processes are executed by synchronising the tools to the moving material web. "Flying saw" and "cross cutter" are techniques widely used in these contexts to increase productivity but usually they are studied from a control point of view.

This work highlights the kinematic and dynamic synthesis of the general framework of a flying machining device with the emphasis on the driving system chosen and the design parameter definition, in order to guarantee the required performance in terms of flexibility and high production volumes. The paper develops and applies a flexibility oriented design to an horizontal wrapping machine.
\end{abstract}

\section{Introduction}

In modern production systems it is increasingly important to increase productivity and at the same time ensure high flexibility levels with respect to the change of product or the size thereof. These requirements are by definition antithetical (Sethi and Sethi, 1990; Shewchuk and Moodie, 1998; Matthews et al., 2006). It is difficult for high production machines to elaborate a range of highly diversified goods. On the other hand it is difficult for flexible machines to reach high production levels.

In most industrial processes, in order to reach high production volumes, the items pass through the production line in a continuous way. Thus the items or process are rarely stopped and all the machining processes are executed with the items in movement. Therefore the tools have to be synchronised to the moving material web and after the machining process, those tools have to be positioned at the starting point for the next cycle.

Processes such as welding, embossing, printing, cutting, sealing, gripping, etc., normally found in a production line, are by their very nature not continuous. In these cases the manufacturing processes have to be executed when the item is stopped. Thus the production line works in an intermit- tently way. To eliminate the wasting of time in stopping and restarting the line it is necessary that the tools follow the items.

Regardless of the industrial field, when the tool moves along a rectilinear trajectory, the application is generally called "flying saws" (Diekmann and Luchtefeld, 2008) and the tool is mounted on a slide that moves together with the piece to be worked. After the machining process has been completed, the tool returns to its original position ready for the next work cycle. Alternatively if the tool moves along a closed trajectory, usually a circular one, the flying tool is referred to as a cross cutter (Diekmann and Luchtefeld, 2008). These kind of manufacturing processes are generally referred to as "flying machining" and several devices have been developed to perform these in various industrial fields.

Regardless of the industrial sector and the flying machining solution chosen, the design set of problems and the methods of controlling the system are the same. As shown in Strada et al. (2012) in fact flying saw and cross cutter systems could been parametrized and studied in an analogous way. Obviously technical solutions developed to move the tools are different but the methodology to synthesise the system could be considered similar. 
It is possible to find several papers that have been published regarding flying machining but none of these deals with the problem in general: each one regards specific cases. Most of them are about the control problem. In Varvatsoulakis (2009), for example, a new digital control system has been designed and implemented in order to replace the existing obsolete one in a cutting system into a production line of STAHL-37 steel tubes. In this case the existing hardware of the cutting system (motor, drive, mechanical equipment) has been maintained. A similar approach is presented in Bebic et al. (2012), but in this case the authors suggest substituting the drive and control systems in order to improve the performance of the cross cuter in the paper or board production line. For these purposes a close examination of the characteristics and requirements of basic subsystems of the paper-board cross cutter from the control system perspective is done.

The control system is studied in depth in Wu et al. (2014). In that work the authors propose a control architecture based on ARM and FPGA to reach high-speed, high-precision, high dynamic, high rigidity performance in a flyng shear cutting system. In view of the increase in demand in the face of the increasing of wrapper machine request for wrapping machines, particularly in the Chinese market ( $\mathrm{Wu}, 2010)$, the authors of the paper Shao et al. (2012) show a synchronizing servo motion and an iterative learning control useful for horizontal flow wrapper. Also in this case the focus of the work is on the control system and on the architecture whereby one can obtain good cutting accuracy and eliminate the repeatable position error. The control problems have been widely studied since the second half of the last century Shepherd, 1964. With the spread of new electronic devices the control approach changed shifting from analog solutions to digital ones Visvambharan, 1988 up to the more modern approaches mentioned above.

These studies address the control system in reaching the required performance and no analysis is addressed on the layout of the cutting tool. In Peric and Petrovic (1990) an optimal control system is considered in order to minimize the driving torque. In this case kinematic and dynamic are taken into account but without a detailed study on the effects that the design parameters have in terms of attainable productivity. A proposal for the revision of the cross cutter system layout is presented in Hansen et al. (2003). The authors suggest operating the cutter by separately controlled servo drives but, also in this case the focus remains on how to control the cutter position.

This work highlights the kinematic and dynamic synthesis of a general flying machining device. Particular attention is paid to the choose of the driving system and the design parameters, so as to guarantee the required performance in terms of flexibility and high production volumes. By virtue of the generalisation set up in Strada et al. (2012) the design method is refers to the cross cutter solution which is widespread in food packaging systems.
The focus of this study is on a flexibility oriented design procedure which takes into account the input parameters necessary to avoid limitations and constraints to the potential of the machine. A general framework is provided, allowing the designer to assess different possible motor-reducer solutions and design parameter combinations, taking into account the various advantages or limitations in term of flexibility. This new approach satisfies two requirements. The first one can verify, theoretically the cutting flexibility in an existing cutting machine. The second can design a new cutting machine capable of reaching a much higher production flexibility level.

This work is organized as follows. In Sect. 2, the flying cutting machine is described. In Sect. 3 the motion laws adopted to perform the cutting operation are set out and analysed while their effects on the dynamic loads are set out in Sect. 4. In Sect. 5 case study simulations and results are presented. In conclusion in Sect. 6 the final considerations are summarized.

\section{Flow-pack systems: horizontal wrapping machine}

A particularly lively industry in which the flying machining is used is the packaging field. A packaging machine is a system used to cover wholly or partially single items or collected group of them with a flexible material. Wrapping machine is a kind of packaging machine that is used to wrap small items with paper or plastic film. The first noted wrapping machine was developed by William and Henry Rose, in England at the end of the nineteenth century (Hooper, 1999).

The typical layout of a flow-pack machine is depicted in Fig. 1. A specific wrapping machine is taken as an example in order to support the theoretical background with a numeric example. It is worth noting that the following considerations are general and not related to a specific flying cutting technology application. The purpose of this kind of machine is to weld and cut the double plastic film that will form the package, while the product is already between them. The plastic film is unreel by the film feed roller and passes through the forming box that folds it in the final configuration. It is important to note that the product arrives on a conveyor-belt and the plastic film is bent around it. The product moves forward to the unit that package it. A couple of rotating heads are used to execute these operations.

Usually, they are synchronous, having the same motor and control unit, even if some attempts to adopt an asynchronous control strategy have been made (Hansen et al., 2003). On their external circumferences, $n$ tools are mounted with the double purpose to weld and cut the packages. In fact, each tool is constituted by a central saw profile to cut each package, whose ends are simultaneously welded by heat-seals units fitted on the side of the saw profile. 


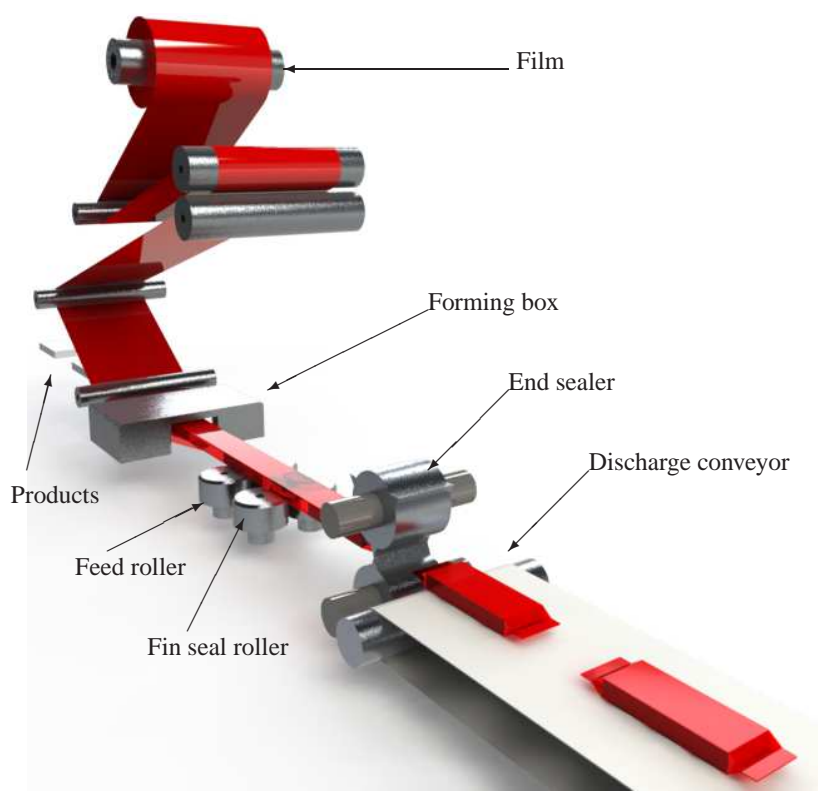

Figure 1. Wrapping machine.

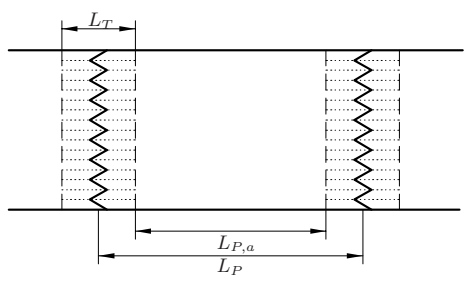

(a) Packaging characteristic dimensions

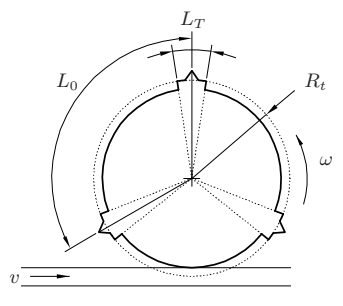

(b) Cutting tool
Figure 2. Characteristic dimensions.

\subsection{Design framework}

Each package is composed by three parts as sketched in Fig. 2a: two welded terminals $\left(L_{T} / 2+L_{T} / 2\right)$ and the central part where the object to package lies $\left(L_{\mathrm{P}, \mathrm{a}}\right)$. It is important to highlight that the expression "product length" $L_{\mathrm{P}}$ used in this work refers to the total length of the packaged unit and not only to the length of the object to be packaged $\left(L_{\mathrm{a}}\right)$.

Thus, the dimensional parameters $L_{T}$ and $L_{\mathrm{P}}$ are the starting dimensions to design the machine.

The more suitable working condition is to have a constant angular speed in order to have negligible dynamic loads and thus this is the nominal working condition. It correspond to an established product length defined as "base" or "design" length $L_{0}$. In every other cases, if the product length is different from the design one, acceleration or deceleration are required in order to account for the imposed target product length. Typically, the base length $L_{0}$ is provided by the costumer because it represents the most common length and thus the target of the designer is to set up a machine which shows the best performance in this configuration.

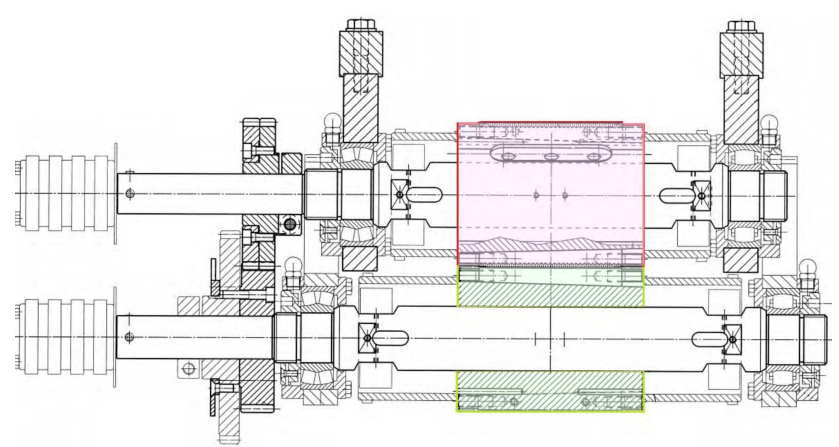

Figure 3. Rotating heads.

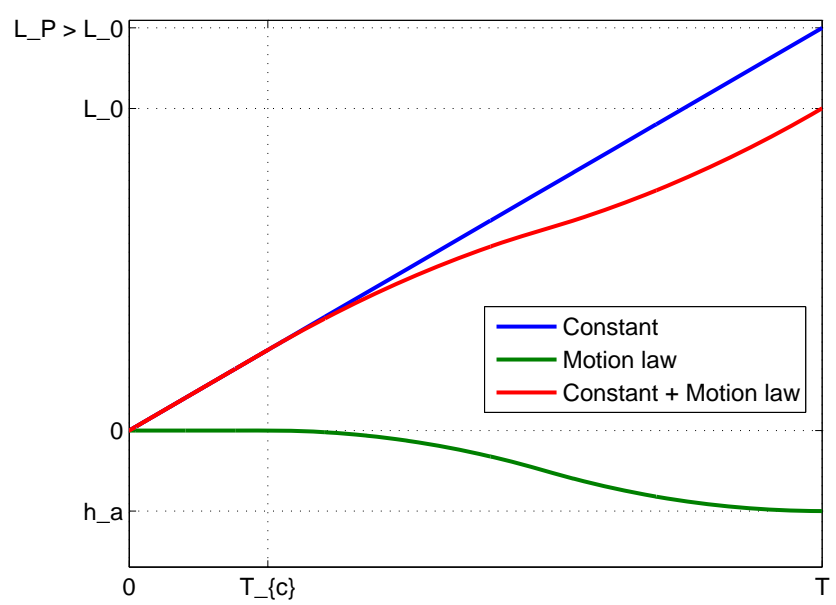

Figure 4. Motion law superposition.

Thus, the radius $R_{t}$ of the rotating head (Fig. 2b) is defined in order to obtain a circumference which length is proportional to the design length itself:

$2 \pi R_{t}=N L_{0}$

The integer ratio $N=2 \pi R_{t} / L_{0}$ corresponds to the number of cutting tools to be installed onto the rotating head. The dimension of the rotating head $R_{t}$ is usually bounded by the layout configuration of the machine (Fig. 3).

\section{Laws of motion}

One of the main characteristics of an automated machine is its productivity: it represents the starting point to define the kinematic link between each part of the whole mechanism. To satisfy the assigned productivity $P$ of a product with a length $L_{\mathrm{P}}$, the conveyor-belt has to maintain a constant velocity $v$ equal to:

$v=\frac{L_{p} \cdot P}{60}$ 

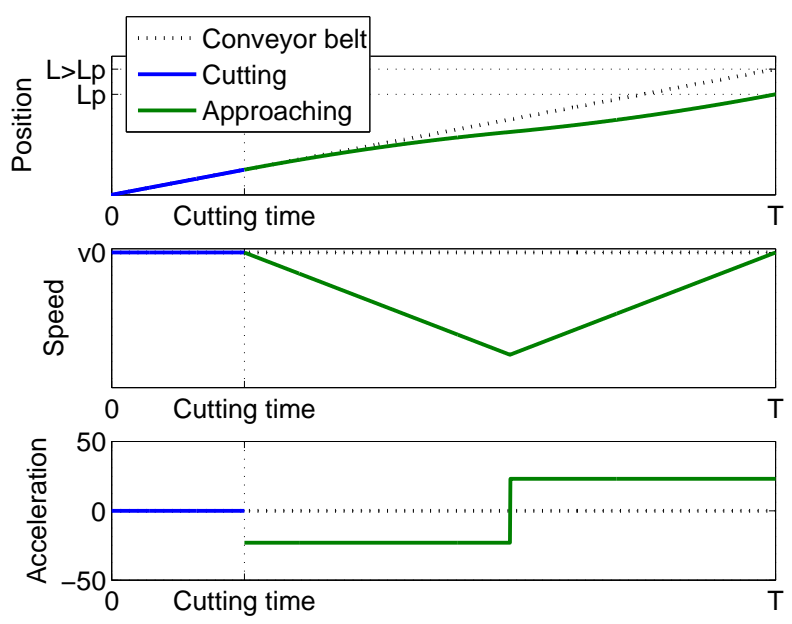

(a)

Figure 5. Motion sequence of the sealing process $\left(L_{\mathrm{P}} \gtrless L_{0}\right)$.

$P$ is usually expressed in pieces $\min ^{-1}$, the cycle time is equal to $T=60 / P$. The total time $T$ is defined as the sum of the duration of two phases:

$T=T_{t}+T_{\mathrm{a}}$

- the cutting phase $T_{t}$. It is the part of the cycle dedicated to weld and cut the packaging of the product,

- the approaching phase between two cuts. It corresponds to the time $T_{\mathrm{a}}$ from the finish of a cut and the beginning of the following one.

\subsection{Cutting phase}

During the cutting phase the angular velocity of the rotating head is kept constant to cut and weld the packaging properly. The tangential velocity of the rotating head has to be equal to the one of the conveyor-belt $v$, resulting in a null relative velocity between them. Thus, the conveyor-belt velocity $v$ can be also defined as:

$v=\frac{L_{t}}{T_{t}}$

because during the cutting phase $T_{t}$ the conveyor-belt shift of a distance equal to $L_{t}$. This condition allows to define the angular velocity $\omega_{t}$ of the rotating head during the cutting phase:

$\omega_{t}=\frac{v}{R_{t}}=\frac{L_{t}}{R_{t} T_{t}}$
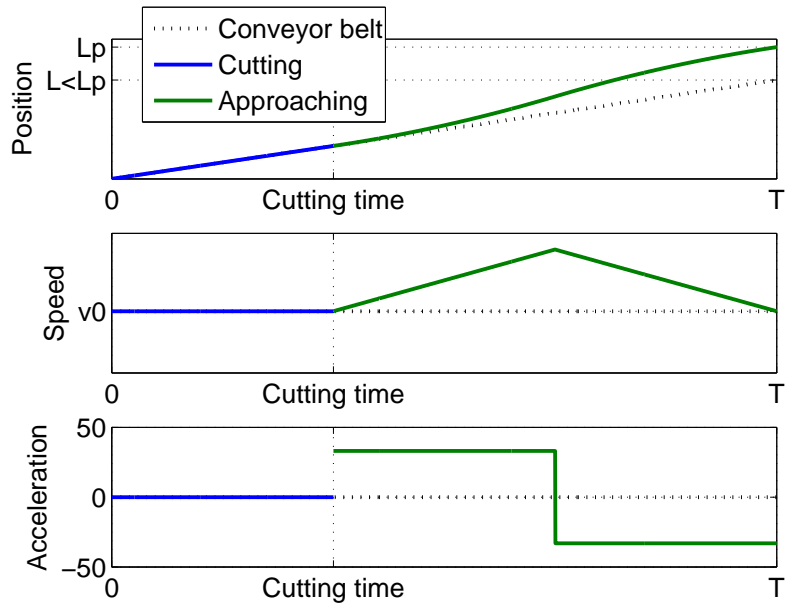

(b)

Usually the length of the welded part of the packaging is defined a-priori and does not depend to the product length $L_{\mathrm{P}}$. Thus, the length $L_{t}$ is not a design parameter for the law of motion because it is imposed by the dimension of the cutting tools and it is typically defined by the costumer.

\subsection{Approaching phase}

As mentioned above, if the product length is equal to the design one $\left(L_{\mathrm{P}}=L_{0}\right)$ the rotating head maintains during the approaching phase a constant angular velocity $\omega_{\mathrm{a}}$ equal to the one of the cutting phase $\left(\omega_{t}\right)$. If the product length is greater or smaller than the design one, the angular velocity of the rotating head during the approaching phase must decrease or increase to properly repositioning the tool for the next cutting phase. A smart approach to generalize the problem is to describe the angular velocity $\omega$ as the sum of two contributes:

- $\omega_{t}$ : the constant angular velocity that allows to cut the $L_{0}$ length,

- $\omega_{\mathrm{a}}=\omega_{t}+\Delta \omega$ where $\Delta \omega$ the variation of angular velocity needed to get the tools in the correct position to execute the next cut.

The variation of the angular speed $\Delta \omega$ depends on the product length and the design length. It is null only if the product length is equal to the design one. In the other cases to define its value it can be convenient to consider the equivalent linear path of the tool as a function of time. Using this different point of view it is possible to define the law of motion of the tool as the superimposition of the path corresponding to the constant angular speed and of the " $\Delta$ " path needed to reach at the correct position and time the package to process 
(Fig. 4), considering that its duration is equal to the one of the approaching phase one and it correspond to a linear distance equal to $h_{\mathrm{a}}=L_{0}-L_{t}$.

Two conditions can be reached:

- $L_{\mathrm{P}}<L_{0}$. The approaching length $h_{\mathrm{a}}$ is greater than the required one: $h=L_{\mathrm{P}}-L_{t}$. The rotating heads must accelerate to recover this additional length.

- $L_{\mathrm{P}}>L_{0}$. The approaching length $h_{\mathrm{a}}$ is smaller than the required one: the rotating heads must decelerate. In extreme cases, it must rest or reverse the rotation direction.

A motion law with a total lift equal to $h=L_{\mathrm{P}}-L_{t}$ and a duration time equal to $T_{\mathrm{a}}=T-T_{t}$ is adopted to perform the modulation of the rotating heads velocity. In Fig. 5 both the cases above described are shown. The dotted line represents the feed of the conveyor-belt. Being its speed constant, as a function of time, it has a linear trend, starting from zero and ending at the processed length $L_{\mathrm{P}}$. During the cutting phase, the feed of the rotating heads is the same of the one of the conveyor-belt, being null the relative velocity between them. If the product length is longer than the design one, the rotating head have to slow down (Fig. 5a). If it is smaller than the design one, the rotating head must increase its angular velocity in order to recover the length deficit as reported in Fig. 5b.

\subsection{Dimension-less design of motion laws}

Named $y(t)$ the path of the rotating head during the approaching phase, it is important to note that its "shape" is not defined a priori. In fact, some different laws of motion, even if they result in very similar behavior in the positioning, differ in relevant ways if the corresponding accelerations are analyzed as shown for three different motion laws in Fig. 6.

Each law of motion can be expressed using a dimensionless space and time parameters:

$\zeta=\frac{y(t)}{h} \xi=\frac{t}{T_{\mathrm{a}}}$

The results is that the law of motion is totally describable using the corresponding $\zeta=\zeta(\xi)$ function, with $0 \leq \zeta \leq 1$ and $0 \leq \xi \leq 1$. The velocity and the acceleration are obtainable using the following differential relations:

$\dot{y}=\frac{\mathrm{d} y}{\mathrm{~d} t}=\frac{\mathrm{d}(h \zeta)}{\mathrm{d}\left(T_{\mathrm{a}} \xi\right)}=\zeta^{\prime} \frac{h}{T_{\mathrm{a}}}$

$\ddot{y}=\frac{\mathrm{d} \dot{y}}{\mathrm{~d} t}=\frac{h}{T_{\mathrm{a}}} \frac{\mathrm{d} \zeta^{\prime}}{\mathrm{d} t}=\frac{h}{T_{\mathrm{a}}} \frac{\partial \zeta^{\prime}}{\partial \xi} \frac{\mathrm{d} \xi}{\mathrm{d} t}=\zeta^{\prime \prime} \frac{h}{T_{\mathrm{a}}^{2}}$

being $\zeta^{\prime}$ and $\zeta^{\prime \prime}$ the dimensionless expressions of velocity and acceleration, respectively.

Every law of motion must satisfy null speed both at the starting and at the ending time instants $\left(\zeta^{\prime}(\xi=0)=\zeta^{\prime}(\xi=\right.$
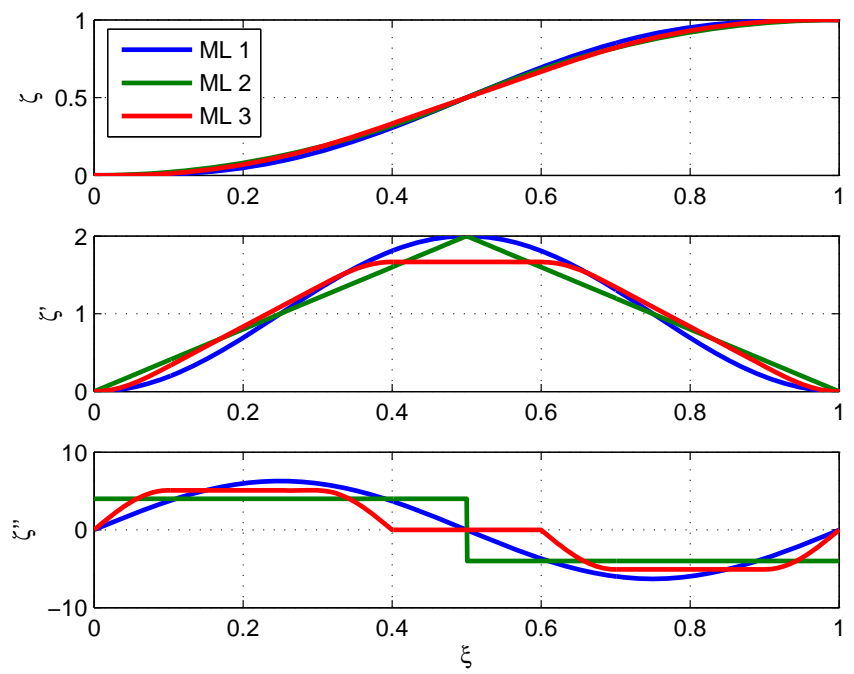

Figure 6. Comparison between different motion laws.
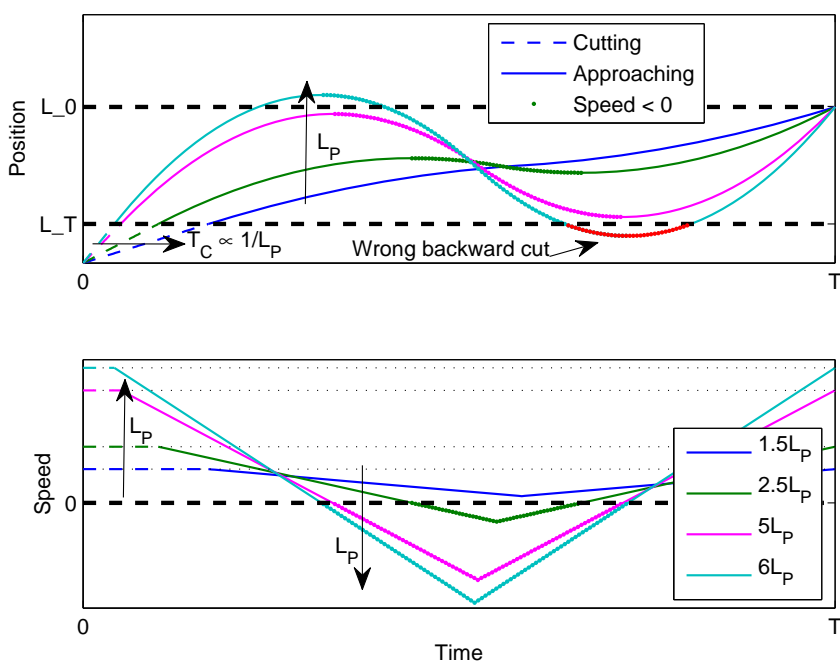

Figure 7. Backward cut increasing the product length.

$1)=0$ ), while it must provide the correct lift starting from $\zeta(\xi=0)=0$ reaching $\zeta(\xi=1)=1$ at the end. As a consequence, it can be demonstrated that the only constrains on the dimensionless acceleration $\zeta^{\prime \prime}$ are:

$\int_{0}^{1} \zeta^{\prime \prime}(\xi) \mathrm{d} \xi=0 \quad \int_{0}^{1} \zeta^{\prime \prime}(\xi) \zeta d \xi=-1$

Using the dimensionless form to describe the laws of motion, some coefficients can be defined to capture several of their notable properties. Thus, it is possible to define the dimension-less speed coefficients $C_{\mathrm{v}}$, that is useful to take into account the peak value of the speed, defined as:

$C_{\mathrm{v}}=\dot{y}_{\max } \frac{h}{t_{\mathrm{a}}}$ 


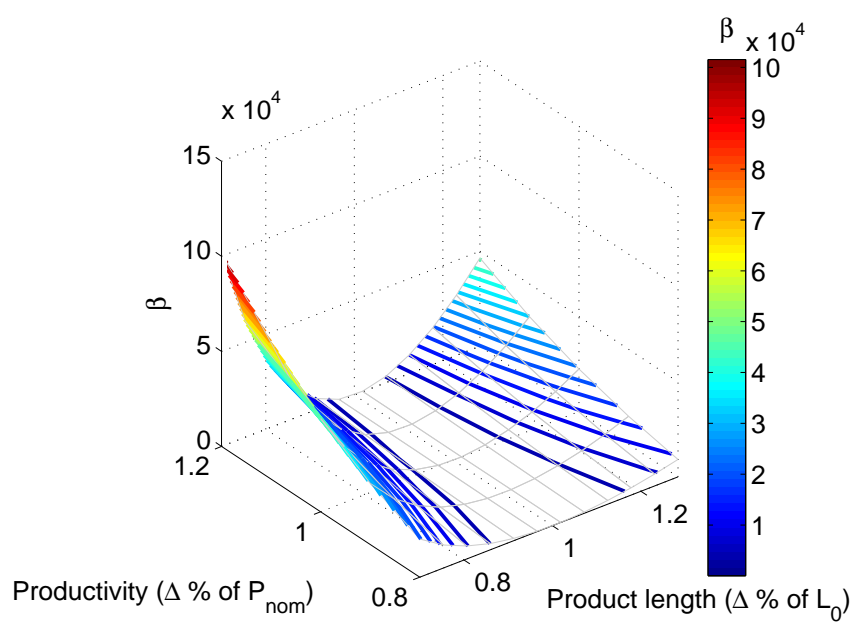

(a)

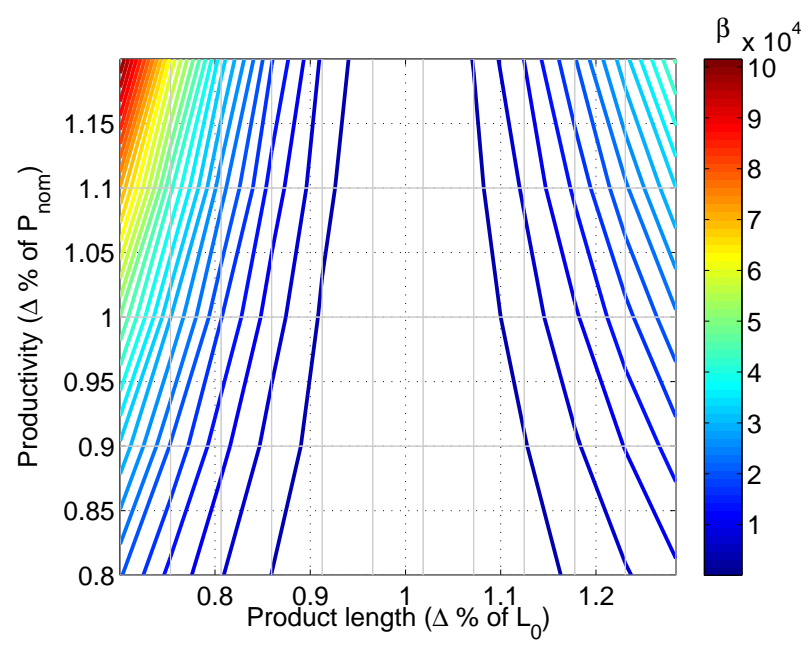

(b)

Figure 8. $\beta$ as a function of product length and productivity.

Table 1. Dimension-less r.m.s. acceleration and speed coefficients.

\begin{tabular}{lrrr}
\hline Motion law & $C_{\mathrm{a}}$ & $C_{\mathrm{a}, \mathrm{rms}}$ & $C_{v}$ \\
\hline Acc const symm & 4 & 4 & 2 \\
$1 / 3-1 / 3-1 / 3$ & 4.5 & 3.67 & 1.5 \\
Cubic & 6 & 3.46 & 1.5 \\
Cycloidal & $2 \pi$ & 4.44 & 2
\end{tabular}

Furthermore, dealing with acceleration, it is possible to define the dimension-less acceleration coefficient $C_{\mathrm{a}}$ and the dimension-less root mean square (r.m.s.) acceleration coefficient $C_{\mathrm{a}, \mathrm{rms}}$ defined respectively as:

$C_{\mathrm{a}}=\ddot{y}_{\max } \frac{h}{t_{\mathrm{a}}^{2}} C_{\mathrm{a}, \mathrm{rms}}=\ddot{y}_{\mathrm{rms}} \frac{h}{t_{\mathrm{a}}^{2}}$

A comparative collection of $C_{\mathrm{a}}, C_{\mathrm{a}, \mathrm{rms}}$ and $C_{\mathrm{v}}$ is provided (Table 1) in order to highlight their effectiveness in describing and comparing the properties of different laws of motion.

The advantage of using the dimension-less form to deal with the different laws of motion is that they are quickly comparable referring to the coefficients that summarize their performance. As an example, using the dimensional-less coefficients $C_{\mathrm{a}, \mathrm{rms}}$, it is possible to highlight the role of the adopted law of motion on the root-mean-square value of the angular acceleration of the rotating heads:

$\dot{\omega}_{L, \mathrm{rms}}=\frac{a_{\mathrm{rms}}}{R_{T}}=\frac{C_{\mathrm{a}, \mathrm{rms}}}{R_{T}} \frac{h}{T_{\mathrm{a}}^{2}} \frac{T_{\mathrm{a}}}{T}$

being $a_{\mathrm{rms}}$ the tangential acceleration of the rotating head, calculated on the whole duration time $T$ while the dimensionless coefficient refers only to the approaching phase $T_{\mathrm{a}}$.

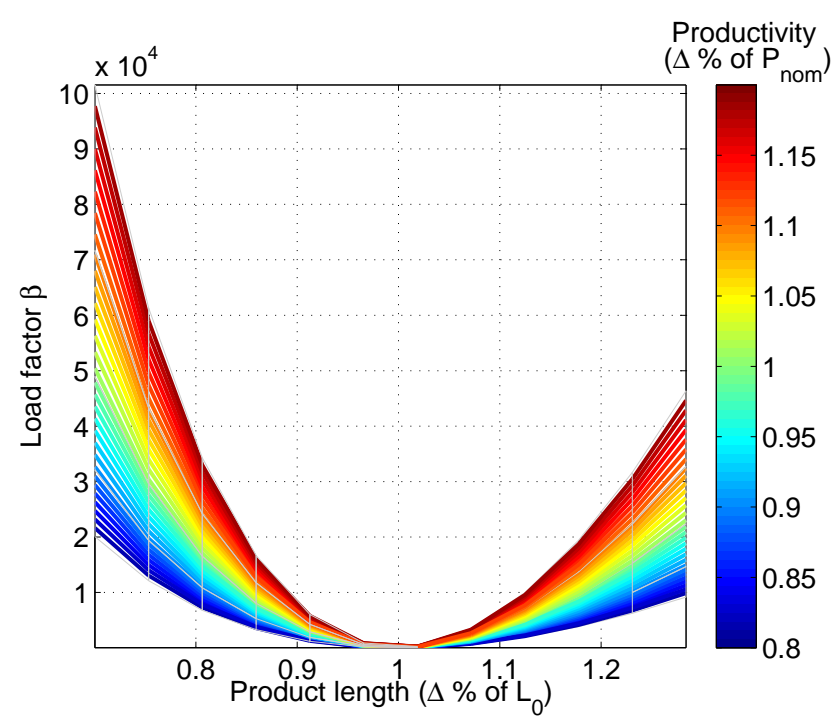

Figure 9. $\beta$ as a function of product length.

\section{Dynamics analysis}

The sizing of the motor-reducer unit is performed under the hypothesis of pure inertial load considering that during the cutting phases, both the friction and the cutting forces are negligible. With this assumption, the only load that the motor have to face with is the rotating heads own inertial load.

\section{1 $\alpha-\beta$ method}

To properly size the motor-reducer unit, the $\alpha-\beta$ method is adopted (Giberti et al., 2011, 2010). This method has the advantage of highlighting and separate the terms of the power 


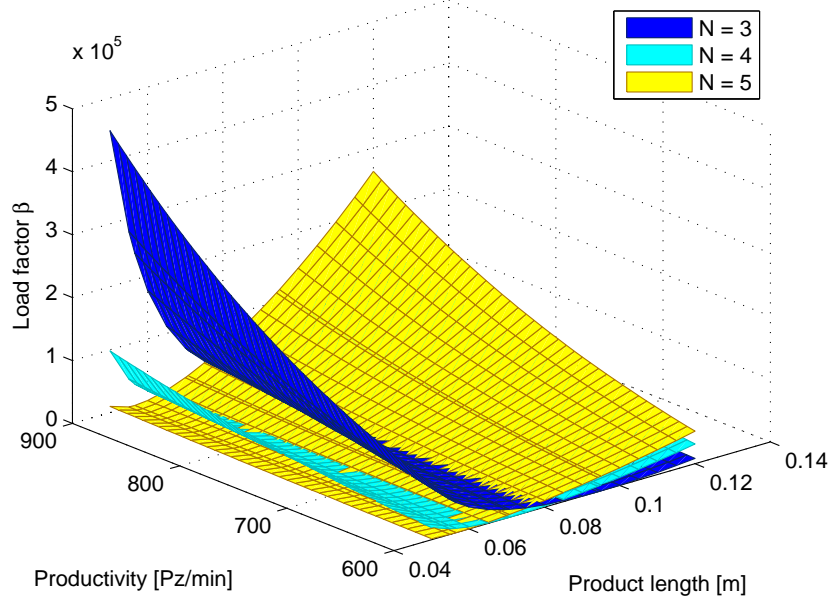

Figure 10. $\beta$ surface as a function of tools number.

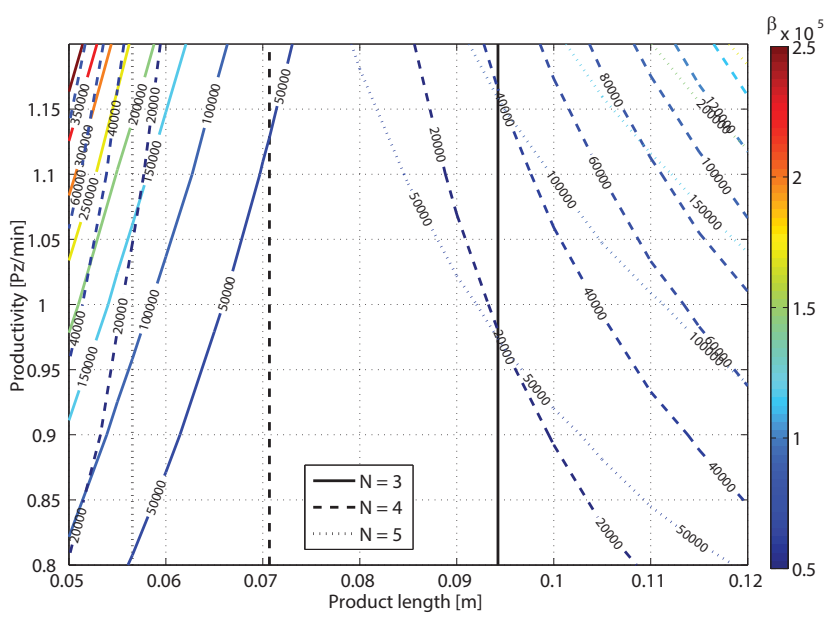

Figure 11. $\beta$ as a function of input parameters.

balance that regards the motor unit and the reducer. This method allows both to avoid an iterative design procedure and to define, for each motor unit considered, the corresponding range of transmission ratios that are suitable for the analyzed application. The motor performance is described by a key-factor called accelerating factor $\alpha$, defined as the ratio between the square of the nominal torque of the motor $C_{\mathrm{m}}$ and its own rotational inertial momentum $J_{\mathrm{m}}$ :

$\alpha=\frac{C_{\mathrm{m}}^{2}}{J_{\mathrm{m}}}$

The accelerating factor derives from the rated motor torque condition $C_{\mathrm{m}, \mathrm{rms}}<=C_{\mathrm{m}}$ used to check the motor thermal equilibrium in which the value $C_{\mathrm{m}, \mathrm{rms}}$ is the root mean square of the torque required by the motor to carry out the task. This is calculated by:

$C_{\mathrm{m}, \mathrm{rms}}=\int_{0}^{t_{\mathrm{a}}} \frac{1}{t_{\mathrm{a}}}\left(\tau C_{\mathrm{r}}+J_{\mathrm{m}} \frac{\dot{\omega}_{\mathrm{r}}}{\tau}\right)^{2} \mathrm{~d} t$

where $t_{\mathrm{a}}$ is the cycle time, $J_{\mathrm{m}}$ the rotor inertia, $\tau$ the transmission ratio and $C_{\mathrm{r}}$ and $\dot{\omega}_{\mathrm{r}}$ the load torque and the load angular acceleration respectively. Substituting the square of $C_{\mathrm{m}, \mathrm{rms}}$ into the rated motor torque condition it is possible to solve the inequality with respect to the accelerating factor term defined beforehand.

A more refined definition of the accelerating factor is the specific accelerating factor that is described in Giberti et al. (2014), but for the aims of this work the simpler one presented above has been considered sufficiently accurate. The load factor $\beta$ contains the information regarding the root mean square load during a cycle and thus it allows to summarize in one single parameter the load the motor is subject to (using a thermal design criterion):

$\beta=2\left(\dot{\omega}_{\mathrm{r}, \mathrm{q}} C_{\mathrm{r}, \mathrm{q}}^{*}+\overline{\dot{\omega}_{r} C_{\mathrm{r}}^{*}}\right)$

where $\dot{\omega}_{\mathrm{r}, \mathrm{q}}$ and $C_{\mathrm{r}, \mathrm{q}}^{*}$ are, respectively, the root mean square of the angular acceleration and the resistant torque while $\overline{\dot{\omega}_{r} C_{\mathrm{r}}^{*}}$ is the mean value of their product. Having defined both $\alpha$ and $\beta$, the condition for the correct sizing of motor-reducer unit can be re-written (Giberti et al., 2011) as:

$\alpha \geq \beta+f(\tau)$

being $\tau$ the transmission ratio defined as:

$\tau=\frac{\omega_{\mathrm{r}}}{\omega_{\mathrm{m}}}$.

The load factor $\beta$ is directly linked to the flexibility of the machine. It is equal to zero only if the product length $L_{\mathrm{P}}$ is equal to the design length $L_{0}$, while, as shown in Sect. 3, it grows if the rotating head needs to be accelerated or decelerated to process a greater or a product length smaller than the base one.

\subsection{Load factor}

As a consequence of pure inertial load assumption, the load factor $\beta$ defined in Eq. (4) becomes:

$\beta=4 J_{L} \dot{\omega}_{\mathrm{r}, \mathrm{rms}}^{2}$

being $J_{L}$ the momentum of inertia of the couple of rotating heads $\left(J_{L}\left(R_{t}\right)=2 J_{T}\left(R_{t}\right)\right)$. It is important to highlight 
Table 2. Test case value.

\begin{tabular}{lll}
\hline Parameter & Value & unit \\
\hline$R_{t}$ & $=0.045$ & {$[\mathrm{~m}]$} \\
$L_{T}$ & $=0.0236$ & {$[\mathrm{~m}]$} \\
$P$ & $=750$ & {$\left[\mathrm{pz} \mathrm{min}{ }^{-1}\right]$} \\
$L_{0}$ & $=0.0942$ & {$[\mathrm{~m}]$} \\
$J_{T}$ & $=0.0126$ & {$\left[\mathrm{~kg} \mathrm{~m}^{2}\right]$} \\
$C_{\mathrm{a}, \mathrm{rms}}$ & $=3.67$ & {$[-]$} \\
$C_{\mathrm{v}}$ & $=1.5$ & {$[-]$} \\
\hline
\end{tabular}

that the best operative condition corresponds to $\beta$ equal to zero, that implies no inertial loads, obtainable only with a null $\dot{\omega}_{\mathrm{r}, \mathrm{rms}}$.

Combining Eqs. (7) and (1), it is possible to highlight the design terms:

$\beta=4 J_{L}\left[\frac{C_{\mathrm{a}, \mathrm{rms}}}{R_{T}} \frac{h}{t_{\mathrm{a}}^{2}} \frac{t_{\mathrm{a}}}{T}\right]^{2}=4 J_{L}\left[\frac{C_{\mathrm{a}, \mathrm{rms}}}{R_{T}} L_{\mathrm{P}} \frac{\frac{2 \pi R_{t}}{N}-L_{\mathrm{P}}}{L_{\mathrm{P}}-L_{T}}\left(\frac{P}{60}\right)^{2}\right]^{2}$

This Eq. (8) is particularly important because it allows to describe the load factor as a function of the input parameters.

\subsection{Gearbox}

The change in transmission ratio range, defined as $\Delta \tau=$ $\tau_{\max }-\tau_{\min }$, can also be expressed as a function of the input parameters:

$\Delta \tau=\sqrt{J_{\mathrm{m}}} \frac{\sqrt{\alpha} \pm \sqrt{\alpha-4 J_{\mathrm{r}} \dot{\omega}_{\mathrm{r}, \mathrm{rms}}}}{2 J_{\mathrm{r}} \dot{\omega}_{\mathrm{r}, \mathrm{rms}}}=\tau_{\mathrm{opt}}\left[\sqrt{\frac{\alpha}{\beta}} \pm \sqrt{\frac{\alpha}{\beta}-1}\right]$

being the ratio $\sqrt{J_{\mathrm{m}} / J_{\mathrm{r}}}$ the optimal transmission ratio $\tau_{\text {opt }}$. It is worth noting that this equation is function only of the load factor and not of the single input parameters of which it is function. Thus it represents a general result for every input parameters combinations which produces the same value of $\beta$.

Finally, the last check on the available $\tau$ serves the purpose of ensuring that the maximum angular velocity required by the law of motion can be provided. Using the introduced formulation:

$$
\begin{aligned}
& \omega_{\max }=\omega_{\mathrm{cost}}+\Delta \omega=\frac{v}{R_{t}}+\frac{v_{\max }}{R_{t}}= \\
& =\frac{P \cdot 2 \pi}{60 N}+\frac{C_{\mathrm{v}}}{R_{t}} \frac{h}{t_{\mathrm{a}}}=\frac{P \cdot 2 \pi}{60 N}\left[1+C_{\mathrm{v}} \frac{L_{o}-L_{p}}{L_{\mathrm{P}}-L_{t}}\right]
\end{aligned}
$$

where $C_{\mathrm{v}}$ is the dimension-less velocity coefficient depending to the specific law of motion (Table 1) adopted and referring only to the approaching phase.

\section{Numerical analysis and results}

In the previous section, the load factor $\beta$ and the admissible range for the transmission ratio $\Delta \tau=\tau_{\max }-\tau_{\min }$ were

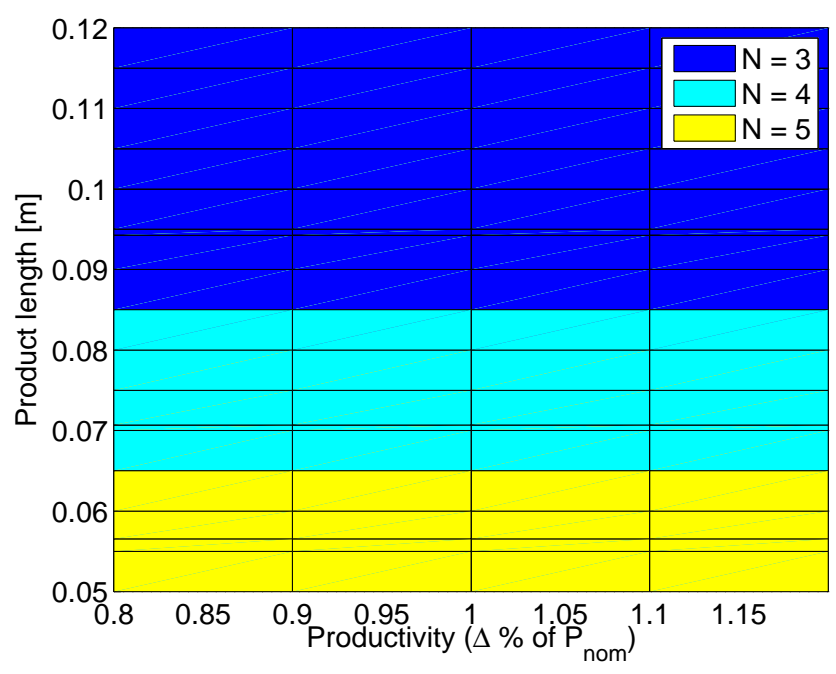

Figure 12. $\beta$ surface as a function of tools number - bottom view.

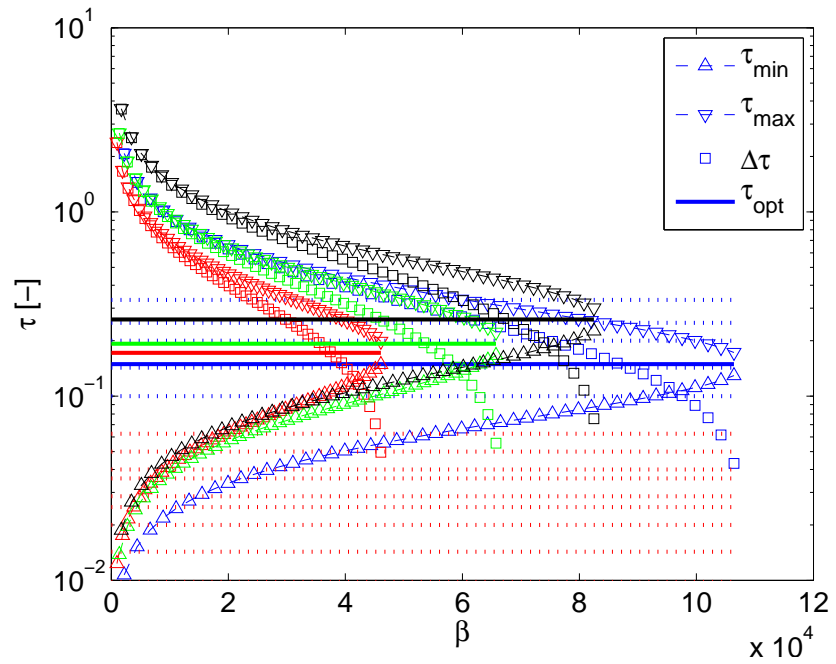

Figure 13. $\tau$ as a function of load factor $\beta$ for four different motors

expressed as a function of the input parameters. Numerical results are obtained in this section for a real wrapping machine with the parameters shown in Table 2.

It is worth underling that the acceleration and the deceleration of the driving law of motion are equal and constant (motion law labeled "Acc const symm" in Table 1). No refinements have been made on the law of motion because the aim of this work is to investigate its role on the flexibility of the machine and not comparing different adoptable solutions. Nonetheless, it is important to highlight that in some cases the selected law of motion is not able to perform the desired operation. In fact, due to a product length larger than the design one, an erroneous cut could be done if the tool happens to move backward too much as reported in Fig. 7. This fact results in a maximum product length processable using the adopted law of motion. This constraint can be avoided using 
a different law of motion that implies the block of the tool. In the presented results this condition was reached in order to avoid introducing a complication not useful to the aim of the present work.

As an example, Fig. 8a reports the surface that graphically describes the load factor as a function of both the variation in the productivity $P$ and the product length $L_{\mathrm{P}}$. For sake of clarity, Fig. 8 reports the top view of the 3-D surface.

It could be seen that if the product length is equal to the design one the load factor is still equal to zero irrespective of the assigned productivity. It is worth noting that the growing of the load factor as a consequence of the change of the product length is not symmetrical. In Fig. 9, is shown that $\beta$ is more sensitive to a decrease of the product size instead of its increase.

Finally, the load factor is more sensitive to a growth in productivity than in a change of product length. Furthermore, the productivity of high-speed automated lines is defined as the one of the so called bottleneck workstation that is the station with the lowest nominal production rate Liberopoulos and Tsarouhas, 2005. As a consequence, the effect of the product length on the productivity not only affect the single machine but involves the whole automated line productivity and should be carefully taken into account by designers.

A refinement of the analysis consists in considering the effect of the number of cutting tools $N$. The results is a group of surfaces (Fig. 10) that represent the functions:

$\beta=f\left(L_{\mathrm{P}}, P, N\right)$

with different values of input parameters. A top view is reported in Fig. 11.

This kind of comparative plot can be used to properly design the machine using the a simple procedure:

- identify the number of tools $N$ in order to obtain the smallest load factor to package with a certain length and with an assigned productivity (Fig. 12).

- design the most flexible flying-cutting machine minimizing the curvature of the surface corresponding to a certain number of tools. In the presented application, the smoothest surface is obtained with $N$ equal to 4 tools. The solution corresponding to $N$ equal to 3 shows unsuitable high value corresponding to the combination of high productivity and short product length.

- define the maximum productivity allowed with a prescribed set of input parameters. The load factor grows as the third power of $P$ and, as a consequence, high values of $\beta$ could be quickly reached. Changing the number of tools allows to obtain a larger productivity than the one reachable without changing the rotating heads setup.

Finally, a collection of $\beta-\tau$ plots is presented in Fig. 13. It is important to underline that this kind of plot depends only on the selected motor unit $(\alpha)$ and on the load factor $(\beta)$ but not to the single input parameters resulting in a more general point of view of the problem. This last plot gives two advices to the designer. The first is that, the bigger the load factor becomes, the smaller the range of admissible transmission ratio $\Delta \tau$ is. In the worst condition, corresponding to $\alpha=\beta$, the only useful transmission ratio is the optimum one $\left(\tau_{\mathrm{opt}}\right)$. It also allows to identify the maximum load factor the motor can withstand.

\section{Conclusions}

This paper deals with the flexibility-oriented design of a flying-cutting machine. A general framework is provided, allowing the designer to assess different possible motorreducer solutions and design parameter combinations, taking into account the various advantages or limitations in terms of flexibility. This new approach satisfies two requirements. The first one can verify, theoretically the cutting flexibility in an existing cutting machine. The second can design a new cutting machine capable of reaching a much higher production flexibility level.

A specific wrapping machine is used as example to describe the methodology but this choice does not limit the extendibility of the method to other flying machine layouts.

By means of the $\alpha-\beta$ sizing motor method it has been possible to obtain an expression that highlights the influence on the motor load factor with respect to the machine parameters such as the number of cutting tools installed, the motion law adopted and the size of the product required to be wrapped. Thus it is possible to compare motor-reducer solutions and to select one so as to ensure, on the one hand, larger productivity and, on the other hand, a larger range of product size.

Edited by: J. A. Gallego Sánchez

Reviewed by: R. Sato and one anonymous referee

\section{References}

Bebic, M., Rasic, N., Statkic, S., Ristic, L., Jevtic, D., Mihailovic, I., and Jeftenic, B.: Drives and control system for paper-board cross cutter, 15th International Power Electronics and Motion Control Conference and Exposition, EPE-PEMC 2012 ECCE Europe, art. no. 6397495, LS6c.31-LS6c.38, 2012.

Diekmann, A. and Luchtefeld, K.: Drive Solutions, Mechatronics for Production and Logistics, in: Intermittent drives for cross cutters and flying saws, edited by: Kiel, E., 378-389, SpringerVerlag, Berlin, Heidelberg, 2008.

Giberti, H., Cinquemani, S., and Legnani, G.: A practical approach to the selection of the motor-reducer unit in electric drive system, Mech. Based Des. Struc., 39, 303-319, 2011.

Giberti, H., Cinquemani, S., and Legnani, G.: Effects of Transmission Mechanical Characteristics on the Choice of a MotorReducer, Mechatronics, 20, 604-610, 2010. 
Giberti, H., Clerici, A., and Cinquemani, S.: Specific Accelerating Factor: One More Tool in Motor Sizing Projects, Mechatronics, 24, 898-905, 2014.

Hansen, D., Holtz, J., and Kennel, R.: Cutter distance sensors for an adaptive position/torque control in cross cutters, IEEE Ind. Appl. Mag., 9, 33-39, 2003.

Hooper, J. H.: Confectionery Packaging Equipment, Springer, Gaithersburg, Maryland, 1999.

Liberopoulos, G. and Tsarouhas, P.: Reliability analysis of an automated pizza production line, J. Food Eng., 69, 79-96, 2005.

Matthews, J., Singh, B., Mullineux, G., and Medland, T.: Constraint-based approach to investigate the process flexibility of food processing equipment, Comput. Ind. Eng., 51, 809-820, 2006.

Peric, N. and Petrovic, I.: Flying Shear Control System, IEEE T. Ind. Appl., 26, 1049-1056, 1990.

Sethi, A. K. and Sethi, S. P.: Flexibility in manufacturing a survey, Int. J. Flex. manuf. Sys., 2, 289-328, 1990.

Shao, W., Chi, R., and Yu, L.: Synchronizing servo motion and iterative learning control for automatic high speed horizontal flow wrapper, Proceedings of the 2012 24th Chinese Control and Decision Conference, CCDC, art. no. 6244470, 2994-2998, 2012.
Shepherd, R.: A Computer-Controlled Flying Shear, Students' Quarterly Journal, 34, 143-148, doi:10.1049/sqj.1964.0006, 1964.

Shewchuk, J. P. and Moodie, C. L.: Definition and classification of manufacturing flexibility types and measure, Int. J. Flex. manuf. Sys., 10, 325-349, 1998.

Strada, R., Zappa, B., and Giberti, H.: An unified design procedure for flying machining operations, ASME 2012 11th Biennial Conference on Engineering Systems Design and Analysis, ESDA 2012, 2, 333-342, 2012.

Varvatsoulakis, M. N.: Design and implementation issues of a control system for rotary saw cutting, Control Eng. Pract., 17, 198202, 2009.

Visvambharan, B. B.: On-line digital control system for a flying saw cutting machine in tube mills, Industrial Electronics Society, IECON '88. Proceedings, 14 Annual Conference of IEEE Industrial Electronics, vol. 2, 385-390, doi:10.1109/IECON.1988.665170, 1988.

Wu, H., Wang, C., and Zhang, C.: Design of Servo Controller for Flying Shear Machine Based on ARM and FPGA, J. Netw., = 9, 3038-3045, 2014.

Wu, Y.: China Packaging Machinery Industry is Facing Tremendous Challenges, China Food Industry, 183, 6 pp., 2010. 